\title{
Differences in the Impact of Pneumococcal Serotype Replacement in Individuals With and Without Underlying Medical Conditions
}

\author{
Daniel M. Weinberger, ${ }^{1}$ Joshua L. Warren, ${ }^{2}$ Tine Dalby, ${ }^{3}$ Eugene D. Shapiro, ${ }^{1,4}$ Palle Valentiner-Branth, ${ }^{5}$ Hans-Christian Slotved, ${ }^{3}$ and \\ Zitta Barrella Harboe ${ }^{3,6}$
}

Departments of ${ }^{1}$ Epidemiology of Microbial Diseases and ${ }^{2}$ Biostatistics, Yale School of Public Health, New Haven, Connecticut; ${ }^{3}$ Department of Bacteria, Parasites and Fungi, Statens Serum Institut, Copenhagen, Denmark; ${ }^{4}$ Department of Pediatrics, Yale School of Medicine, New Haven, Connecticut; and Infectious Disease Epidemiology and Prevention, Statens Serum Institut, and ${ }^{6}$ Department of Infectious Diseases and Pulmonary Medicine, Nordsjaellands Hospital, Copenhagen University Hospital, Denmark

Background. Pneumococcal conjugate vaccines (PCVs) have had a well-documented impact on the incidence of invasive pneumococcal disease (IPD). However, declines in IPD due to vaccine-targeted serotypes have been partially offset by increases in IPD due to nonvaccine serotypes (NVTs). The goal of this study was to quantify serotype-specific changes in the incidence of IPD that occurred in different age groups, with or without certain comorbidities, following the introduction of 7-valent pneumococcal conjugate vaccine (PCV7) and 13-valent pneumococcal conjugate vaccine (PCV13) in the childhood vaccination program in Denmark.

Methods. We used nationwide surveillance data for IPD and a hierarchical Bayesian regression framework to estimate changes in the incidence of IPD associated with the introduction of PCV7 (2007) and PCV13 (2010) while controlling for serotype-specific epidemic cycles and unrelated secular trends.

Results. Following the introduction of PCV7 and PCV13 in children, the net impact of serotype replacement varied considerably by age group and comorbidities. Differences in the magnitude of serotype replacement were due to variations in the incidence of NVTs in the different risk groups before the introduction of PCVs. The relative increases in the incidence of IPD caused by specific NVTs did not differ appreciably between risk groups in the postvaccination period. Serotype replacement offset a greater proportion of the benefit of PCVs in strata in which the NVTs comprised a larger proportion of cases prior to the introduction of the vaccines.

Conclusions. These findings could help to predict the impact of next-generation PCVs in specific risk groups.

Keywords. pneumococcus; serotype replacement; conjugate vaccines; Bayesian hierarchical model.

Pneumococcal conjugate vaccines (PCVs) have had a well-documented impact on the incidence of invasive pneumococcal disease (IPD) worldwide [1-5]. Because PCVs interrupt transmission of pneumococcus among healthy children, the introduction of PCVs has led to reductions in the incidence of IPD among both vaccinated children and (indirectly) among unvaccinated adults $[1,6]$. A meta-analysis of data from the United States, Europe, and Australia found that, on average, IPD due to serotypes in the 7-valent PCV (PCV7) declined by approximately $90 \%$ among adults [1]. However, these declines in IPD due to vaccine-targeted serotypes (VTs) were at least partially offset by increases in IPD caused by nonvaccine serotypes (NVTs), a phenomenon known as serotype replacement [1, 7]. The net benefit of PCVs is therefore determined by both

Received 7 May 2018; editorial decision 2 October 2018; accepted 8 October 2018; published online October 15, 2018.

Correspondence: D. M. Weinberger, Department of Epidemiology of Microbial Diseases, Yale School of Public Health, PO BOX 208034, New Haven, CT 06520 (daniel.weinberger@yale.edu). Clinical Infectious Diseases ${ }^{\circledR} \quad$ 2019;69(1):100-6

(C) The Author(s) 2018. Published by Oxford University Press for the Infectious Diseases Society of America. All rights reserved. For permissions, e-mail: journals.permissions@oup.com. DOI: 10.1093/cid/ciy875 the magnitude of the decline in the incidence of VT-IPD and by increases in the incidence of NVT-IPD. The net benefit of PCVs can vary by risk groups and between populations $[1,8]$. Variations in the net benefit of PCVs between countries can be explained by variations in the serotype distribution of nasopharyngeal carriage among children in the prevaccine period [9]. However, it is not clear why the net benefit of PCVs vary within the same population where exposure is more consistent between risk groups. Understanding variations in the net benefit of PCVs against IPD in different risk groups is important as PCVs with expanded numbers of serotypes are developed and deployed [10].

The goal of this study was to quantify and explain changes in the incidence of IPD caused by VTs and NVTs after the introduction of PCVs in children among different groups of patients in Denmark according to their age and presence of underlying diseases. Distinguishing changes in the incidence of IPD due to the effects by PCVs from those due to unrelated secular trends is a major challenge because of the well-recognized multiyear, serotype-specific epidemic cycles $[11,12]$. We used an analytical framework that estimates and controls for the epidemic cycles of individual serotypes and can estimate serotype- and 
risk group-specific estimates of vaccine effects against IPD as well as average estimates across all serotypes and risk groups.

\section{METHODS}

\section{Data Sources}

Data were retrieved by linking several Danish national registers from 1994 to 2015. The data on cases of IPD (ie, meningitis, bacteremia, and other sterile foci) were obtained from a laboratory-based nationwide surveillance system described elsewhere [13]. The serotype is determined for $>97 \%$ of all IPD isolates from Denmark at the Statens Serum Institut. While surveillance data are available for many decades, data have been recorded more consistently across the registries since 1994 (the start date for this study).

PCV7 was introduced in the Danish childhood immunization program in 2007 with high uptake, and then replaced by 13-valent PCV (PCV13) in 2010 [13]. Throughout the manuscript, "PCV7 serotypes" refers to serotypes 4, 6B, 9V, 14, 18C, $19 \mathrm{~F}$, and 23F, and "PCV13 serotypes" refers to the PCV7 serotypes plus serotypes 1, 3, 5, 6A, 7F, and 19A. All other serotypes were classified as non-PCV7/13 serotypes (NVTs). Prior to 2006-2007, serotype 6C isolates were not identifiable and would have been misclassified as serotype 6A [14]. One hundred two cases $(0.5 \%)$ had data on the serogroup but not the serotype. We performed simple imputation to assign serotypes to these cases (Supplementary Data).

Information on comorbidities was obtained from the Danish National Patient Registry $[15,16]$, a nationwide hospitalization database. The Danish Personal Identification Number was used to link to the IPD data for all cases with the hospitalization database. Comorbidity was defined on the basis of discharge codes based on the International Classification of Diseases, Tenth Revision. We searched for conditions that increase the risk of IPD as defined by the Advisory Committee on Immunization Practices [17], with codes related to chronic heart disease, chronic pulmonary disease, diabetes, alcoholism, liver disease, cerebrospinal fluid leaks, cochlear implants, anatomic or functional asplenia, human immunodeficiency virus and other immunodeficiencies, renal disease, hematological and solid malignancies, rheumatological diseases, and organ transplantation. For the specific codes included in the comorbidity analysis, see the Supplementary Data. A case was defined as belonging to a high-risk group if the patient had a record of any of the indicated conditions at the time of diagnosis with IPD (including if the comorbidity was identified at the time of diagnosis). Due to small sample size, we simply classified individuals as having any of these comorbidities vs none and were not able to evaluate specific comorbidities or combinations.

We estimated population size for each age group, comorbidity level, and year. Population size in each age group and year were obtained from Statistics Denmark [18]. Information on comorbidities was obtained from a random sample of 386612 records from the Danish National Patient Registry, extracted for a different study. We calculated the proportion of these control individuals in each age group and calendar year who had any of the high-risk conditions. Multiplying the proportion of individuals with comorbid diseases in each year and age group by the corresponding population size estimate for the year and age group gave an estimate of the number of individuals in year and stratum.

\section{Model}

Many serotypes exhibit multiyear epidemic cycles and trends unrelated to the vaccine [11]. To account for these patterns when estimating changes associated with the vaccine, we used a hierarchical Poisson model fitted in the Bayesian setting. There were 74 serotypes included in the analysis, and the data were stratified by epidemiological year (July-June) into 5 age groups $(<5,5-17,18-39,40-64$, and $\geq 65$ years $)$ and by comorbidity status (with or without known comorbidities prior to the IPD episode). The outcome variable was the number of IPD cases in each epidemiological year and each stratum. The offset term was the estimated population size in the corresponding stratum in that year. An observation-level random intercept captured overdispersion (ie, unexplained variability) of the count data and could account for short-term events (eg, outbreaks, influenza epidemics) that are not part of a longer-term cycle or trend. We controlled for repeating epidemic cycles using harmonic terms, where the length of the period for each serotype was estimated from the data. Prevaccine trends and changes from the prevaccine trend were captured using linear splines with 3 segments (prevaccine, post-PCV7, and post-PCV13). Knots were placed at the time of introduction of PCV7 and PCV13 to allow for changes in slope at that time associated with PCV introduction. The slopes were estimated hierarchically-estimates for a particular time series were centered around estimates for the serotype, which were, in turn, centered around either a trend for all serotypes (prevaccine trend) or trends for the groups of PCV7, PCV13, or NVTs for the postvaccine trends.

A major assumption of this model is that any serotype-specific trends or cycles that occurred during the prevaccine period would have continued in the postvaccine period. By projecting forward the prevaccine trend and cycles, we calculated a counterfactual estimate for how many cases would have occurred in each year for each serotype and stratum in the absence of vaccination. Comparing the model-fitted estimate and the counterfactual estimates, we calculated the relative change at each postvaccine time point as well as the number of cases prevented. The models were fit using the jags program in R statistical software (https://www.r-project.org/) [19, 20]. Details of the model structure, fitting procedures, and calculation of vaccine effects can be found in the Supplementary Methods. Up-to-date jags code, along with observed and expected model fits, can be found at https://github.com/weinbergerlab/Danish-serotype. 


\section{RESULTS}

\section{Characteristics of Data}

We included 19638 cases of IPD that occurred between July 1994 and June 2015 in Denmark. The proportion of cases that were aged $\geq 65$ years increased from $52 \%$ in $1994-1995$ to $64 \%$ in 2014-2015. Likewise, among the age group $\geq 65$ years, the proportion of cases with at least 1 recorded comorbidity increased steadily from $34 \%$ in $1994-1995$ to $56 \%$ in $2000-2001$ and $74 \%$ in 2014-2015. Of the individuals with a comorbidity, $39 \%$ had multiple comorbidities; multiple comorbidities were more common among $\geq 65$-year-olds (42\%) compared with 18 to 39 -year-olds (24\%). The incidence of IPD declined during the pre-PCV period, with much of this decline occurring during the first couple years of the study period. Several serotypes had downward trends in the pre-PCV period $(3,6 \mathrm{~A}, 12 \mathrm{~F})$, and these were projected to continue declining without PCV introduction (Supplementary Figure 7). When aggregating over all serotypes, some of these patterns are obscured (Figure 1).

IPD caused by several serotypes had significant multiyear epidemic patterns that were independent of the introduction of PCVs. Epidemic cycles were notable for serotypes 1 (7.7 years; 95\% credible interval [CI], 7.4-8.1 years) and 18C (9.7 years; 95\% CI, 8.9-10.0 years) (Supplementary Figure 5). The intensity (amplitude) of the IPD epidemic cycles did not differ significantly by risk group. There was evidence of less-pronounced harmonic variations in the incidence of IPD due to other serotypes (eg, 4, 7F) (Supplementary Figure 5).

\section{Overall Changes in IPD Associated With the Introduction of PCV7 and PCV13}

Considering all age and risk groups together, the PCV7 serotypes have declined gradually and have been nearly eliminated as causes of IPD after the introduction of PCV7, declining by $95 \%$ between 2007-2008 and 2014-2015 (Figure 1). The incidence of IPD due to the 6 additional serotypes in PCV13 increased modestly (36\% increase) between 2007 and 2009, along with other non-PCV7 serotypes, and then declined following the introduction of PCV13 in 2010. The incidence of IPD due to NVTs has increased steadily since the introduction of PCV7, having increased by $50 \%$ since 2007-2008 (Figure 1). These decreases in IPD due to VTs and increases in IPD due to NVTs resulted in a small net decline in the incidence of IPD between 2007 and 2009 and more substantial declines since the introduction of PCV13 (Table 1). Among individual serotypes, IPD due to serotypes 14 and 19F had the greatest absolute declines in incidence, while serotype 8, an NVT, emerged as the leading cause of IPD over the past several years (Figure 2). The incidence of IPD due to serotypes 6C, 10A, 10B, $12 \mathrm{~F}, 15 \mathrm{~A}, 16 \mathrm{~F}, 22 \mathrm{~F}, 23 \mathrm{~B}$, and $24 \mathrm{~F}$ was also significantly higher by 2014-2015 than would have been expected without introduction of the vaccine (Supplementary Figure 2). The incidence of IPD due to serotype 19A increased by $>3.5$-fold following introduction of PCV7, but returned to levels similar to or below baseline following the introduction of PCV13 (Figure 2).

\section{Importance of Serotype Replacement by Age and Comorbidity Level}

By 2014-2015 (4 years post-PCV13), there were substantial net declines in the incidence of IPD in all age groups. Incidence declined by $70 \%$ (95\% CI, $57 \%-78 \%)$ among children $<5$ years of age during this period. In contrast, the incidence of IPD among adults $\geq 65$ years of age with comorbidities declined by just $26 \%$ (95\% CI, 13\%-37\%) (Table 1; Figure 3). Serotype replacement offset the declines in the incidence of IPD due to VTs, but the net effect of these increases varied by age group and those with or without comorbidity. Among children $<5$ years of age, serotype
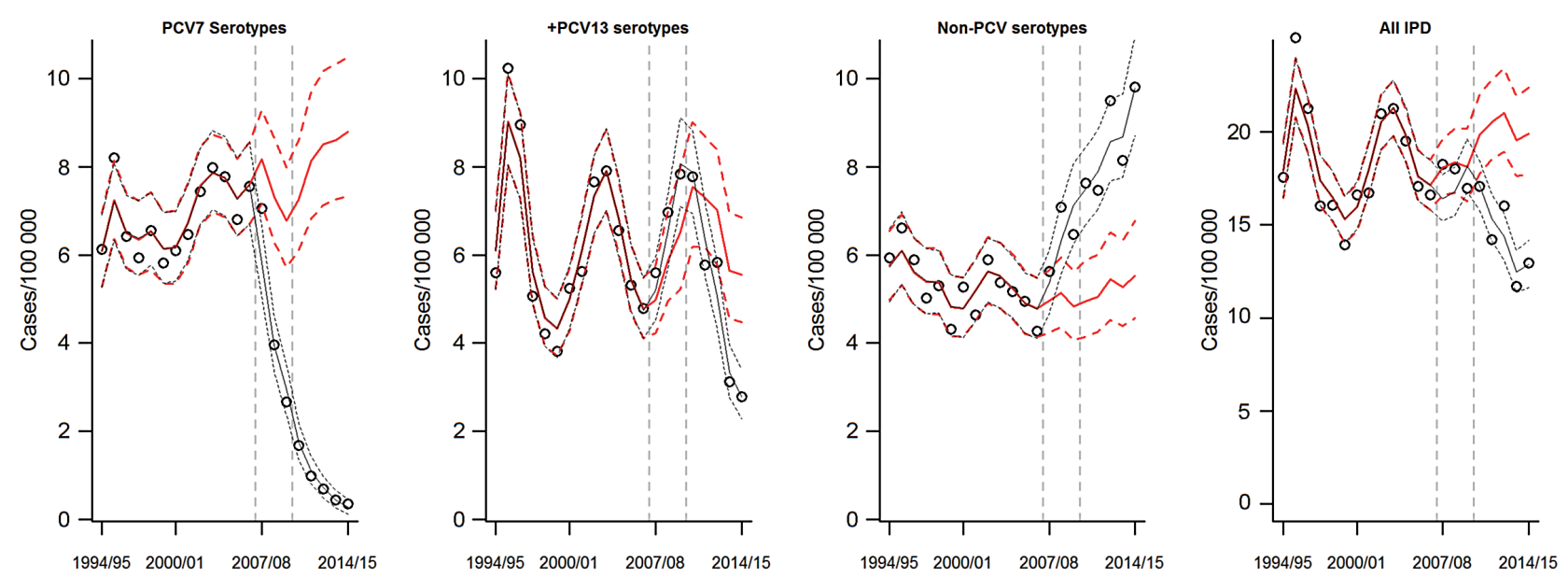

Figure 1. Changes in the incidence of invasive pneumococcal disease (cases/100 000) from 1994-1995 to 2014-2015 for serotypes targeted by 7-valent pneumococcal conjugate vaccine, for the 6 additional serotypes in 13-valent pneumococcal conjugate vaccine (PCV13), for non-PCV13 serotypes, and for all cases combined. The dots represent observed values, the black line $\pm 95 \%$ predictive intervals indicates the model-fitted values, and the red line $\pm 95 \%$ predictive credible intervals represents the estimate of what the incidence would have been in the absence of vaccine. Abbreviations: IPD, invasive pneumococcal disease; PCV, pneumococcal conjugate vaccine; PCV7, 7-valent pneumococcal conjugate vaccine; PCV13, 13-valent pneumococcal conjugate vaccine. 
Table 1. Changes in Invasive Pneumococcal Disease Incidence Caused by 7-Valent and 13-Valent Pneumococcal Conjugate Vaccine Serotypes and Nonvaccine Serotypes by Age Presence of Comorbidity for 2014-2015 Compared With Values Expected in the Absence of Vaccination

\begin{tabular}{|c|c|c|c|c|c|}
\hline Age Group & Comorbidities $^{a}$ & $\begin{array}{c}\% \text { of IPD } \\
\text { Preventable by } \\
\text { PCV7/13 }\end{array}$ & $\begin{array}{c}\text { \% Change in IPD by } \\
\text { PCV13 Serotypes } \\
(95 \% \text { Cl) })^{C}\end{array}$ & $\begin{array}{l}\text { \% Change in IPD by } \\
\text { Nonvaccine } \\
\text { Serotypes }(95 \% \mathrm{CI})^{\mathrm{C}}\end{array}$ & $\begin{array}{c}\text { Net \% Change } \\
(95 \% \mathrm{Cl})\end{array}$ \\
\hline \multirow[t]{2}{*}{$<5 y$} & N & 92 & $-94(-97$ to -91$)$ & $178(60-331)$ & $-72(-80$ to -59$)$ \\
\hline & Y & 83 & $-92(-96$ to -84$)$ & 99 (19-241) & $-64(-77$ to -44$)$ \\
\hline \multirow[t]{2}{*}{$5-17 y$} & N & 81 & $-73(-84$ to -51$)$ & 57 (-21 to 151$)$ & $-50(-67$ to -27$)$ \\
\hline & Y & 74 & $-81(-90$ to -66$)$ & $91(13-223)$ & $-49(-67$ to -24$)$ \\
\hline \multirow[t]{2}{*}{$18-39 y$} & N & 74 & $-72(-82$ to -58$)$ & $75(7-167)$ & $-44(-59$ to -25$)$ \\
\hline & Y & 86 & $-83(-90$ to -72$)$ & $51(-2$ to 126$)$ & $-50(-64$ to -31$)$ \\
\hline \multirow[t]{2}{*}{$40-64$ y } & N & 82 & $-70(-78$ to -59$)$ & $103(45-178)$ & $-26(-40$ to -8$)$ \\
\hline & Y & 75 & $-81(-86$ to -73$)$ & $48(14-93)$ & $-37(-49$ to -23$)$ \\
\hline \multirow[t]{2}{*}{$\geq 65 y$} & N & 66 & $-79(-85$ to -72$)$ & $60(19-110)$ & $-44(-54$ to -31$)$ \\
\hline & Y & 70 & $-78(-83$ to -71$)$ & $94(53-144)$ & $-26(-38$ to -12$)$ \\
\hline
\end{tabular}

Abbreviations: $\mathrm{Cl}$, credible interval; IPD, invasive pneumococcal disease; N, not known; PCV7, 7-valent pneumococcal conjugate vaccine; PCV13, 13-valent pneumococcal conjugate vaccine: Y, any comorbidity.

${ }^{a}$ Any comorbidity, as defined by the Advisory Committee on Immunization Practices according to International Classification of Diseases, Tenth Revision, codes registered during and prior to hospitalization for IPD.

${ }^{\text {b} T h e ~ p e r c e n t a g e ~ o f ~ I P D ~ p r e v e n t a b l e ~ b y ~ P C V 7 / 13 ~ i s ~ c a l c u l a t e d ~ u s i n g ~ t h e ~ c o u n t e r f a c t u a l ~ e s t i m a t e s ~ f r o m ~ t h e ~ m o d e l ~ f o r ~ 2014-2015 ~ b y ~ d i v i d i n g ~ t h e ~ v a l u e s ~ f o r ~ P C V 7 / 13 ~ s e r o t y p e s ~ b y ~ t h e ~ o v e r a l l ~}$ counterfactual predicted number of cases.

'The percentage change for both PCV13 serotypes and non-PCV13 serotypes was calculated by comparing the fitted values for 2014-2015 with the counterfactual estimates from the model for 2014-2015, which give an estimate for how many cases would have occurred in the absence of vaccine introduction and expressed as estimated cases with $95 \%$ credible interval.

replacement offset a small amount of the decline in VT-IPD; without replacement, we would have expected to achieve an $86 \%$ reduction in IPD, compared with an estimated $72 \%$ decline (Table 1; Figure 3 ). In contrast, among adults $\geq 65$ years of age with any comorbidities, serotype replacement offset more than half of the reduction in VT-IPD by the end of the period (ie, IPD would have declined by $54 \%$ without serotype replacement but actually declined by $26 \%$ ) (Table 1 ; Figure 3 ).

The importance of serotype replacement was particularly notable following the introduction of PCV7 and prior to the introduction of PCV13. During this time, the IPD incidence among children $<5$ years of age declined substantially despite replacement. However, among older children and young adults, the increase in NVT-IPD completely offset declines in VT-IPD (ie, there was no net change in the overall incidence of IPD) (Supplementary Figure 1).

\section{Differences in the Net Effect of Replacement on IPD Due to Variations in Baseline Incidence}

We next evaluated why the net effect of the vaccine on rates of IPD differed by age group and comorbidity. Such differences

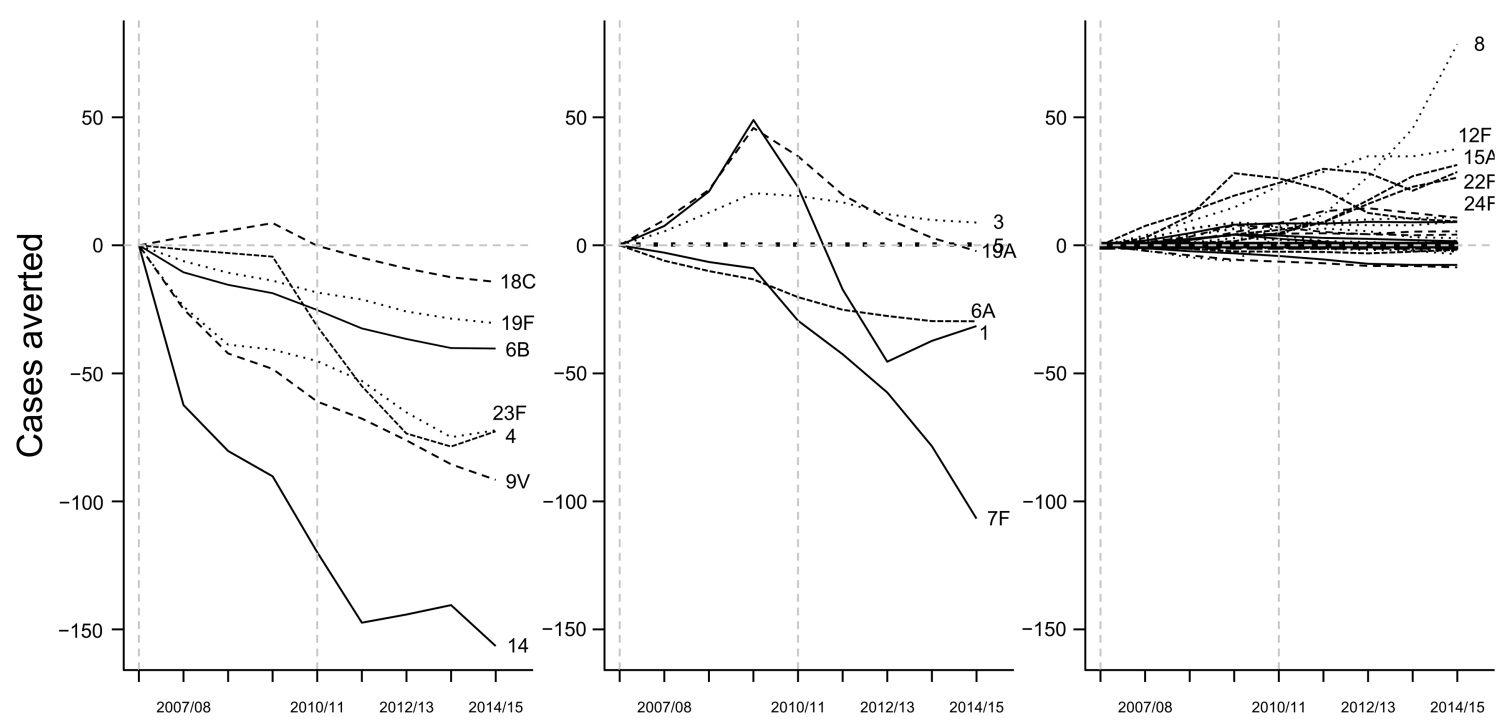

Figure 2. Estimated cases of invasive pneumococcal disease in all age groups prevented by vaccination (negative values) or gained through serotype replacement (positive values) in each year since the introduction of 7-valent/13-valent pneumococcal conjugate vaccine (PCV7/13). Stratified by PCV7-targeted serotypes, PCV13-additional serotypes, and nonvaccine serotypes. The dashed vertical lines indicate the introduction of PCV7 and PCV13. 

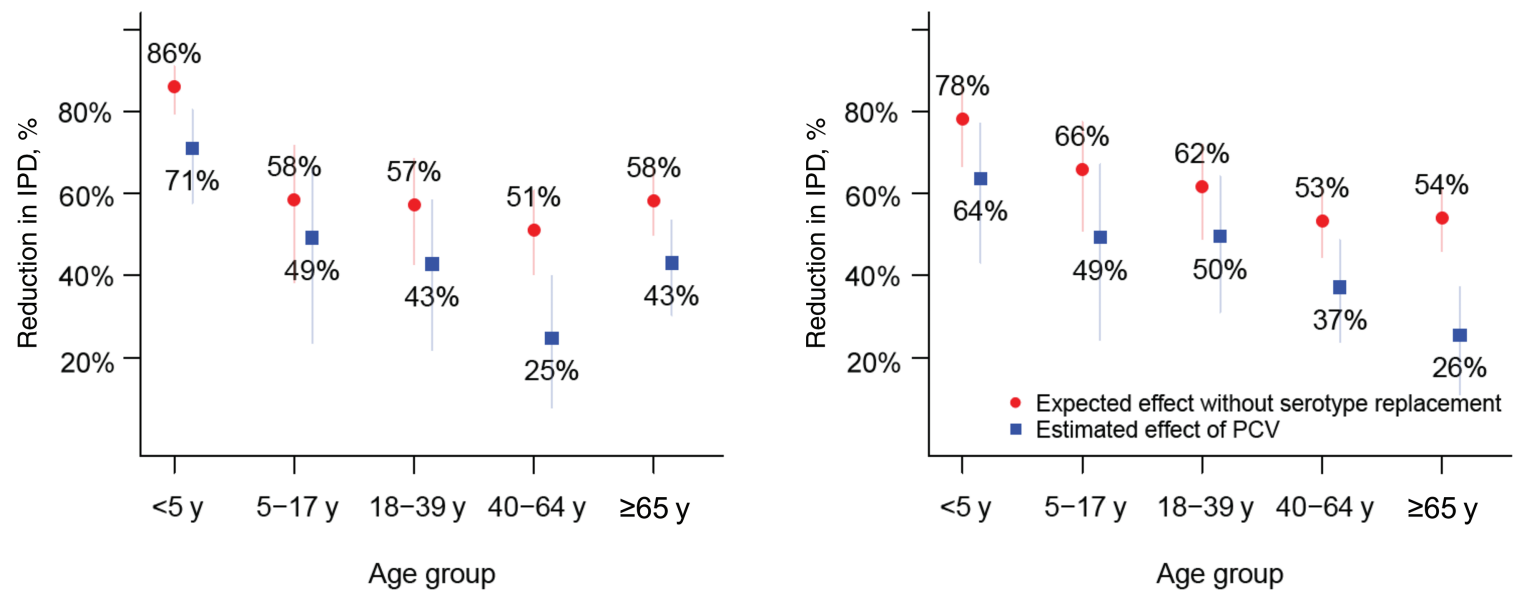

Figure 3. Reduction in invasive pneumococcal disease (IPD) and the effect of serotype replacement, by age group and comorbidity status. The blue dots indicate the actual estimated reduction in the incidence of IPD that results from both declines in vaccine-targeted serotypes and increases in nonvaccine serotypes. The red dots indicate the reduction in the incidence of IPD that would have been expected if there had been no serotype replacement. The distance between the blue and red dots indicates how much serotype replacement reduces the benefit of the vaccine. Bars indicate 95\% credible intervals. Abbreviations: IPD, invasive pneumococcal disease; PCV, pneumococcal conjugate vaccine.

between groups could be due to variations in the prevaccine incidence of IPD due to NVTs relative to VTs, or to the magnitude of the relative increases or decreases. As could be expected, there was a strong association between the proportion of cases of IPD that were due to PCV7/13-targeted serotypes in a given age or comorbidity group prior to the introduction of PCV7/13 and the magnitude of the net decline in the incidence of IPD in that group (Table 1; Supplementary Figures 3 and 4).

The relative increases in the incidence of NVT-IPD were notably similar across age groups (with and without comorbidity) (Table 1; Figure 4). There was modest variability between age/comorbidity groups in the strength of the relative decline in the incidence of IPD due to PCV-targeted serotypes. The declines were
A

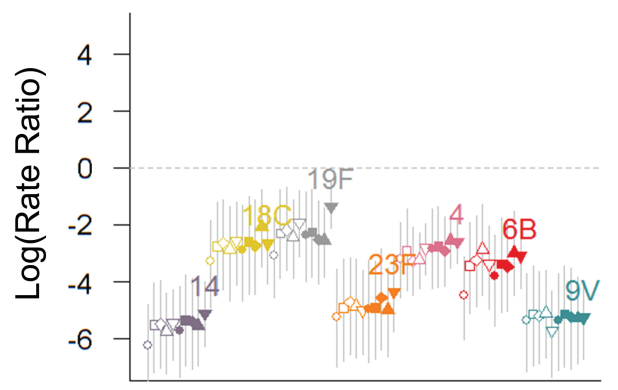

B

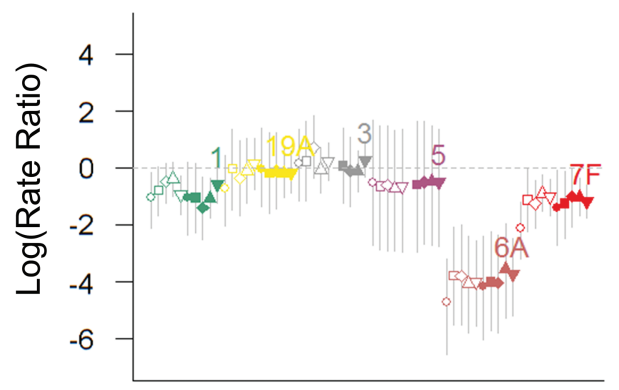

C

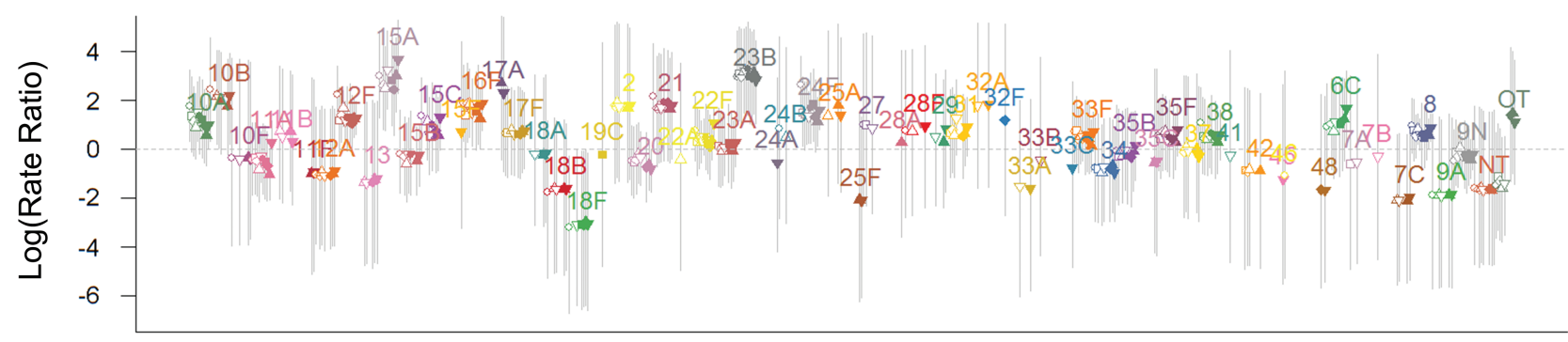

Figure 4. Estimated vaccine-associated change in the incidence of each serotype and risk group for 2014-2015. Log (rate ratios) $\pm 95 \%$ credible intervals. The dots are ordered by age group $(<5,5-17,18-39,40-64, \geq 65$ years) and by comorbidity (open symbols: no comorbidity; closed circles: at least 1 comorbidity). $A$, Seven-valent pneumococcal conjugate vaccine serotypes. $B$, Thirteen-valent pneumococcal conjugate vaccine (PCV13) unique serotypes. C, Non-PCV13 serotypes. 
greatest $(94 \%)$ in children $<5$ years of age (the only age group in which a high proportion of individuals received the vaccine) (Table 1) and with smaller, but substantial (72\%-83\%) declines in other age groups with or without comorbidity (Table 1). Serotype 19F declined by $75 \%$, and the other PCV7 serotypes were effectively eliminated. Because PCV7 might provide cross-protection for serotype $6 \mathrm{~A}$, and PCV13 might provide cross-protection for serotype $6 \mathrm{C}$, we performed sensitivity analyses where we classified serotype $6 \mathrm{~A}$ as a PCV7 serotype and 6C as a PCV13 serotype. This change in classification did not have any notable effect on the serotype-specific or overall estimates (Supplementary Figure 6).

\section{DISCUSSION}

There were clear and substantial declines in the incidence of VT-IPD following the introduction of PCVs in children, as well as smaller but substantial increases in the incidence of NVTIPD. The net effect of these changes varied somewhat by age and comorbidity status. Increases in NVT-IPD offset more of the decline in VT-IPD following the introduction of PCV7 than PCV13. By using a modeling strategy that controlled for the natural epidemic cycles of IPD by the individual serotypes, it was possible to obtain credible estimates for serotype-specific changes associated with the introduction of PCVs in different groups of patients, despite natural fluctuations in the serotype-specific incidence of IPD.

While the impact of PCVs against IPD has been well established for children [1-4], estimates of the impact for adults have varied. For instance, a meta-analysis by Feikin et al [1] found that in some countries (eg, the United States), there were significant declines in the overall incidence of IPD in adults despite replacement. However, in other countries, increases in the incidence of IPD due to non-PCV7 serotypes completely offset declines in IPD due to PCV7-targeted serotypes among adults. Our results could help to explain these patterns. We found that, among adults aged 40-64 years with known comorbidities, serotype replacement following the introduction of PCV7 offset declines in IPD by VTs. Among all age groups, the relative increases in the incidence of NVT-IPD were similar across age and comorbidity levels, and the relative declines in VT-IPD were also similar. Differences in the net effect of the vaccine between groups can therefore be explained by serotype patterns in the period before PCV7 was introduced. In particular, the balance of VTs to NVTs during the pre-PCV period is important. Such variations in IPD serotype distribution could occur due to differential invasiveness of some serotypes in children and adults or due to variations in exposure between populations [21]. In groups in which a large fraction of IPD in the pre-PCV period was due to NVTs, serotype replacement offset a larger fraction of the decline in IPD.

The relative decline in the incidence of IPD due to PCVtargeted serotypes was greatest in children $<5$ years of age. These children benefit both from direct protection by the vaccines against invasive infections and from the decline in exposure to the VTs that results from PCVs reducing carriage of vaccine serotypes. Because effectiveness of the vaccine against targeted serotypes is greater for IPD than for carriage [22,23], we would expect that directly vaccinated individuals would have larger declines in the incidence of IPD due to PCV-targeted serotypes.

Our study has strengths as well as limitations. A strength is that we used data from a comprehensive national surveillance system with a long baseline period, which allows us to estimate secular trends and harmonic variations. Additionally, we used a hierarchical modeling approach that reduces the effect of random variation in the disaggregated data and that allowed us to obtain estimates of the effect of PCVs on the incidence of IPD for different subgroups. A limitation is that we defined comorbidity status based on classification of comorbidities from a national hospitalization register, which might result in misclassification and underestimation of individuals presenting with less-severe comorbid disease, particularly if these patients had not been hospitalized prior to the IPD episode. Other factors that are well known to affect susceptibility to IPD and possibly to IPD due to specific serotypes, such as smoking, crowding, socioeconomic status, and previous vaccination with any pneumococcal vaccine, were not included in the analysis. Additionally, due to small sample size, we were unable to evaluate specific comorbidities or to evaluate combinations of comorbidities; we expect that with sufficient sample size, performing these additional stratifications would amplify differences between groups. Finally, we assumed that linear trends continue indefinitely into the postvaccine period. This could result in over- or underestimating declines depending on the direction of the secular trends. Nonparametric or time-series forecasting methods could help to mitigate this issue.

In conclusion, we have demonstrated important increases in the number of cases of IPD caused by NVTs that accompanied declines in cases caused by VTs following the introduction of PCV7 and PCV13 in children. The importance of these changes varied by age and comorbidity group, particularly during the period after introduction of PCV7 and before introduction of PCV13. These detailed findings will help to predict the impact of serotype replacement on IPD in different risk groups following the introduction of next-generation PCVs and could assist the selection of serotypes that should be included in new PCVs for use in adults.

\section{Supplementary Data}

Supplementary materials are available at Clinical Infectious Diseases online. Consisting of data provided by the authors to benefit the reader, the posted materials are not copyedited and are the sole responsibility of the authors, so questions or comments should be addressed to the corresponding author.

\section{Notes}

Disclaimer. The findings and conclusions of this work are those of the authors and do not necessarily represent the official position of the National Institutes of Health (NIH) or the Statens Serum Institut.

Financial support. This work was funded by the National Institute of Allergy and Infectious Diseases, NIH (grant numbers R01AI123208 
and R56AI110449 to D. M. W.). Support was also received from the Bill \& Melinda Gates Foundation (award numbers OPP1176267 and OPP1114733); the National Institute on Aging (grant number P30AG021342; Scholar at the Claude D. Pepper Older Americans Independence Center at Yale University School of Medicine to D. M. W.); and the National Center for Advancing Translational Science, components of NIH and the NIH Roadmap for Medical Research (grant numbers UL1TR001863, UL1 TR000142, and KL2 TR000140).

Potential conflicts of interest. $\quad$ D. M. W. has received consulting fees from Pfizer, Merck, GlaxoSmithKline, and Affinivax. Z. B. H. has received travel grants and consultancy fees from Pfizer and GSK. D. M. W. and Z. B. $\mathrm{H}$. have received support from the Robert Austrian Research Award in Pneumococcal Vaccinology, which is supported by Pfizer. H. C. S. is participating in a Pfizer-sponsored project. All other authors report no potential conflicts. All authors have submitted the ICMJE Form for Disclosure of Potential Conflicts of Interest. Conflicts that the editors consider relevant to the content of the manuscript have been disclosed.

\section{References}

1. Feikin DR, Kagucia EW, Loo JD, et al; Serotype Replacement Study Group. Serotype-specific changes in invasive pneumococcal disease after pneumococcal conjugate vaccine introduction: a pooled analysis of multiple surveillance sites. PLoS Med 2013; 10:e1001517.

2. von Gottberg A, de Gouveia L, Tempia S, et al; GERMS-SA Investigators. Effects of vaccination on invasive pneumococcal disease in South Africa. N Engl J Med 2014; 371:1889-99.

3. Pilishvili T, Lexau C, Farley MM, et al; Active Bacterial Core Surveillance/ Emerging Infections Program Network. Sustained reductions in invasive pneumococcal disease in the era of conjugate vaccine. J Infect Dis 2010; 201:32-41.

4. Harboe ZB, Dalby T, Weinberger DM, et al. Impact of 13-valent pneumococcal conjugate vaccination in invasive pneumococcal disease incidence and mortality. Clin Infect Dis 2014; 59:1066-73.

5. Zhou Z, Deceuninck G, Lefebvre B, De Wals P. Forecasting trends in invasive pneumococcal disease among elderly adults in Quebec. Can J Infect Dis Med Microbiol 2017; 2017:4347206.

6. Savulescu C, Krizova P, Lepoutre A, et al; SpIDnet Group. Effect of high-valency pneumococcal conjugate vaccines on invasive pneumococcal disease in children in SpIDnet countries: an observational multicentre study. Lancet Respir Med 2017; 5:648-56.

7. Weinberger DM, Malley R, Lipsitch M. Serotype replacement in disease after pneumococcal vaccination. Lancet 2011; 378:1962-73.

8. Muhammad RD, Oza-Frank R, Zell E, et al. Epidemiology of invasive pneumococcal disease among high-risk adults since the introduction of pneumococcal conjugate vaccine for children. Clin Infect Dis 2013; 56:e59-67.
9. Flasche S, Le Polain de Waroux O, O’Brien KL, Edmunds WJ. The serotype distribution among healthy carriers before vaccination is essential for predicting the impact of pneumococcal conjugate vaccine on invasive disease. PLoS Comput Biol 2015; 11:e1004173.

10. Caro-Aguilar I, Indrawati L, Kaufhold RM, et al. Immunogenicity differences of a 15-valent pneumococcal polysaccharide conjugate vaccine (PCV15) based on vaccine dose, route of immunization and mouse strain. Vaccine 2017; 35:865-72.

11. Harboe ZB, Benfield TL, Valentiner-Branth P, et al. Temporal trends in invasive pneumococcal disease and pneumococcal serotypes over 7 decades. Clin Infect Dis 2010; 50:329-37.

12. Paulo AC, Sá-Leão R. Periodic cycles of pneumococcal serotypes carried by children before and after 7-valent pneumococcal conjugate vaccine. PLoS One 2017; 12:e0176723.

13. Ingels H, Rasmussen J, Andersen PH, et al; Danish Pneumococcal Surveillance Collaboration Group 2009-2010. Impact of pneumococcal vaccination in Denmark during the first 3 years after PCV introduction in the childhood immunization programme. Vaccine 2012; 30:3944-50. doi:10.1016/j. vaccine.2012.03.060

14. Melnick N, Thompson TA, Beall BW. Serotype-specific typing antisera for pneumococcal serogroup 6 serotypes 6A, 6B, and 6C. J Clin Microbiol 2010; 48:2311-2.

15. Schmidt M, Schmidt SA, Sandegaard JL, Ehrenstein V, Pedersen L, Sørensen HT. The Danish National Patient Registry: a review of content, data quality, and research potential. Clin Epidemiol 2015; 7:449-90.

16. Lynge E, Sandegaard JL, Rebolj M. The Danish National patient register. Scand J Public Health 2011; 39:30-3.

17. Centers for Disease Control and Prevention, Advisory Committee on Immunization Practices. Updated recommendations for prevention of invasive pneumococcal disease among adults using the 23-valent pneumococcal polysaccharide vaccine (PPSV23). MMWR Morb Mortal Wkly Rep 2010; 59:1102-6.

18. Suresh MV, Singh SK, Agrawal A. Interaction of calcium-bound C-reactive protein with fibronectin is controlled by $\mathrm{pH}$ : in vivo implications. J Biol Chem 2004; 279:52552-7.

19. Plummer M. rjags: Bayesian graphical models using MCMC. R package version 3-14. XXX, XXX: XXX, 2014.

20. R Foundation for Statistical Computing. R: a language and environment for statistical computing. Vienna, Austria: R Foundation for Statistical Computing, 2014.

21. Weinberger D, Grant L, Weatherholtz R, Warren J, O’Brien KL, Hammitt LL. Relating pneumococcal carriage among children with disease rates among adults before and after introduction of conjugate vaccines. Am J Epidemiol 2016 83:1055-62.

22. Nicholls TR, Leach AJ, Morris PS. The short-term impact of each primary dose of pneumococcal conjugate vaccine on nasopharyngeal carriage: systematic review and meta-analyses of randomised controlled trials. Vaccine 2016; 34:703-13.

23. Pavia M, Bianco A, Nobile CG, Marinelli P, Angelillo IF. Efficacy of pneumococcal vaccination in children younger than 24 months: a meta-analysis. Pediatrics 2009, 123:e1103-10. 